\title{
Ubik: a multi-agent based simulator for ubiquitous computing applications
}

\author{
Emilio Serrano, Juan A. Botia and Jose M. Cadenas
}

\begin{abstract}
This paper introduces the development of an infrastructure to study highly complex systems of Ambient Intelligence (AmI) which involve a large number of users. The key ideas about the development of a multi-agent based simulation (MABS) for such purposes, Ubik, are given. The paper also extrapolates effective technologies in the development of multi-agent systems (MAS) to the field of MABS. In particular, the basis for the use of forensic analysis as a method to assist the analysis, understanding and debugging of Ubik in particular and the general MABS are set up.
\end{abstract}

Index Terms-Ambient Intelligence, Multi-agent based simulation, Multi-agent systems, forensic analysis

\section{INTRODUCTION}

Ambient Intelligence (AmI) stems from the convergence of ubiquitous computing, ubiquitous communication and intelligent user-friendly interfaces and creates environments that are characterized by their ubiquity, transparency and intelligence[6]. AmI is of great interest to society because in a near future it can help us to do our work, improve our health, do house chores, etc. The research on AmI presents new scientific challenges. Traditional usability engineering methods and tools fail in the development of AmI applications[3]. Established organizations such as the Usability Professional's Organization have recognized the need for new approaches to usability testing[3]. When AmI is applied to a large number of users, there is a point where the real tests are not feasible. This paper tries to provide solutions to these situations.

Multi-agent based simulation, MABS, allow modelers to handle different levels of representation (e.g., "individuals" and "groups", for instance) within an unified conceptual framework. Such versatility makes MABS one of the most favorite and interesting support for the simulation of complex systems [4]. MABS is used in more and more scientific domains [4]: sociology, biology, physics, chemistry, ecology, economy, etc. This paper proposes the use of MABS in AmI. Specifically, complex AmI applications with a large numbers of users are treated. Testing the social behavior of user groups is interesting in these applications. That is, we are interested in the macrosocial perspective. Therefore, the overall objective of our research is to increase the usability of this type of complex AmI applications.

About the field of MABS, generally, researchers are more interested in model design than in model execution and the execution analysis. Specifically, the analysis is viewed as less methodical and hand crafted[4]. The field of Multi

Emilio Serrano, Juan A. Botia and Jose M. Cadenas are with Department of Information and Communications Engineering at the University of Murcia, Spain.

E-mails: \{emilioserra, juanbot, jcadenas\}@um.es
Agent Systems (MAS), a well-established branch of AI, is complementary in several aspects to MABS[2]. MAS theory has scientific approaches to analyze, understand and debug the social level of agents. This paper aims to argue that technologies used in the analysis of MAS can be used for MABS. In particular, forensic analysis is proposed. This technology has already been used successfully to debug MAS in a social level[13]. Forensic analysis is the process of understanding, re-creating, and analyzing arbitrary events that have occurred previously[11].

To achieve the overall objective, increase of the usability of complex AmI applications, four objectives are marked: (1) Developing of an infrastructure to study complex AmI applications. The multi-agent based simulation Ubik achieves this goal. (2) Testing the effectiveness of AmI applications with the infrastructure resulting from the accomplishment of the first goal. This is achieved by extending the Ubik simulation. (3) Development of an infrastructure to assist the analysis, understanding and debugging of group behaviors in general MABS through forensic analysis. (4) Use of the results of the third goal for the simulations resulting in the first two objectives. What we aim is to validate the simulated models, understand them, explain them and, ultimately, achieve the overall objective.

The next section deals with the related work. Then, section III studies in detail the infrastructure for complex AmI applications and the sort of AmI applications which can be studied. Section IV treats the infrastructure for the debugging of MABS. Finally conclusions and future work are given.

\section{RELATED WORKS}

A Living Lab is a laboratory which consists of real life environments where real users together with researchers look together for new services in AmI. In 2000 started first serious plans to build a laboratory that could be used to conduct feasibility and usability studies in AmI[6]. Finally, the HomeLab of Philips was opened on April 24, 2002. Other wellknown living labs are AwareHome Project [7] or Adaptative House[10]. This approach of direct testing in laboratories has proliferated. For example, the European Network of Living Labs is a grown up initiative coming from the own European Living Lab and sponsored by the European Community. On the open living labs $w e b^{1}$ can be found contact information for living labs in 29 countries, only in Spain 19 living labs are joined with this project. However, these approaches are not feasible in applications for thousands of users or more. Research into ubiquitous computing has also used simulations

\footnotetext{
${ }^{1}$ Open living labs website: http://www.openlivinglabs.eu/
} 
such as TATUS [5] and Ubiwise [1] which extend first person shooter games (FPS). However, these simulations, which model the physical environment in a 3D view, do not model the user which is a real player of the game. Besides, they are valid for only a few users and not for thousands of them. Hence, this paper proposes the use of MABS.

Luke et al. [9] introduce the basis of the platform we use to program the simulation Ubik, $\mathrm{MASON}^{2}$, as well as several simulations using it. For example, the simulation Anthrax Propagation in the Human Body has in common with Ubik that treats a field with which you can not experiment with in a laboratory. This simulation, like ours, is useful to help researchers in the field to explore what-if scenarios and to create strategies to attend humans effectively. The MABS have also been used to check the effectiveness of networking applications as we intend. In this way, Network Intrusion is an agent-based model designed to study computer network security[9]. The parameters of the model allow researchers to understand the effects of changes in security policies, just as Ubik does with the AmI applications policies. In general, any work on MABS offers interesting points of our research; the innovation of this paper is the use for AmI applications.

Simulation typically generates huge amounts of data. Analysis of the simulations have not received the attention it deserves. Within MAS theory, we can find literature that deals with the analysis of agents' behavior. In this way, Serrano et al. [13] detail the creation of an infrastructure for forensic analysis for MAS. Forensic analysis is often the basis of most proposals about debugging in MAS and it is often accompanied by comprehensible data representations. There are works that analyze the behavior of the agents groups with petri nets, AUML diagrams, extensions of the propositional dynamic logic, statecharts, dooley graphs, and so on. There are also works that combine forensic analysis and data mining to achieve simple representations of the agent society[14]. Each of these methods to analyze and debug the MAS are directly usable in this field of MABS.

\section{UbiK, A MUlti-AGENT BASED SimUlation For the AMI DOMAIN}

Any AmI facility involves a financial outlay, much larger when AmI is applied in large domains as a skyscraper or even a whole city. For example, we do not know the cost of the HomeLab of Philips [3], a laboratory to study AmI applications, but we are quite sure that is much greater than that needed for the development of any MABS. Simulation analysis helps to verify the effectiveness of AmI applications before installing them. It also allows to check the results of the AmI in diverse and configurable societies, which make these applications more robust. Anyway, the most important application of the presented proposal is using it in AmI applications with so many users that real tests are totally unworkable. For example, HomeLab consist of a house with a living, a kitchen, two bedrooms and a study where these applications cannot be performed.

$\mathrm{Ubik}^{3}$ is an infrastructure to study complex AmI applica-

${ }^{2}$ MASON website: http://cs.gmu.edu/ eclab/projects/mason/

${ }^{3}$ Code, photos and videos of Ubik on: http://ubiksim.sourceforge.net tions which involve a large number of users. It is a MABS programmed in MASON and aims to be as descriptive as possible to be useful for AmI. A longer description implies a more complex process of understanding things of interests for us to explore new technologies to assist the analysis of simulations (section IV). Figure 1 shows Ubik between MASON and AmI applications.

As it will be explained below, figure 1 also shows this proposal is useful using other MABS a part of Ubik. Different MABS frameworks can be used a part of MASON too. And obviously, the assistance in emergencies is not the only interesting AmI application to develop with the presented proposal. Finally, figure 1 shows an Analyzer layer which provides useful resources for the developer in charge of studying the simulations results.

The modeled space in Ubik is treated as an environment compounded by buildings devoted to offices where the main activities are related to working tasks. Regarding the modeled agents, the simulation does not model humans with a cognitive point of view, but reactive. We are interested in human behavior as a member of a group of other humans which, predictably, will have the same behaviors to events of interest. The simulated space and agents must be considered to select valid AmI applications to be studied in Ubik. For example, a scenario in which the human has a reactive behavior is in an emergency. Figure 1 shows this concrete AmI application over Ubik.

The simulated building has a number of floors, stairs, elevators, hallways, rooms (bathrooms, kitchens, resources...), etc. A key idea is that the whole building is configurable, so the user can simulate virtually any scenarios. It is considered that any area without any specific purpose, such as a hallway or room, is a valid work space where an agent can place a table and do its work. More complex structures such as offices or cubicles would require more complex and laborious specifications to set the building, for example, expressed with ontologies. Figure 2 shows the floor of a building simulated with Ubik.

In Ubik, each worker is an agent. The workers have behaviors: staying on the work place, visiting another worker, downing to the street, walking around the office, convening meetings, attending meetings, attending emergency, going to certain rooms and so on. On the other hand, there are different types of workers: managers, subordinates, secretaries, service personnel, and so on. There is a fitness, linked to every worker, which determines the movement velocity. This velocity is lower if the agent is using the stairs. All these factors are configurable to simulate a multitude of societies in Ubik. Figure 2 represents the workers as an oval, more clearly whether they are on the work place, and a line indicates where they are watching.

To study AmI applications in Ubik, first the simulation is extended with the concept of as physical device (i.e. displays, computers, domotic devices, etc.). Then, the business logic is modeled as an AmI application (see figure 1). The effect is that the applications and devices have influence on the simulated space and, indirectly, in the agents. For example, an application of AmI in which the space and the agents are compatible with 


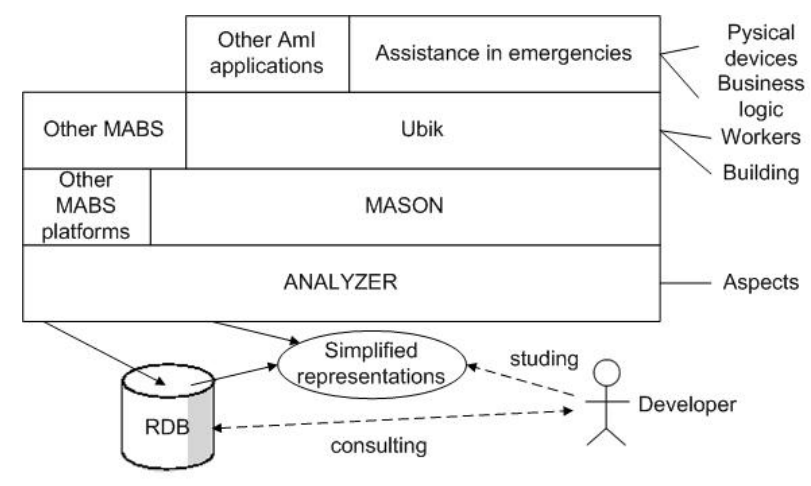

Fig. 1. Proposal of the paper

the Ubik simulation is the assistance in emergencies. With regard to the building, it is required to add sensors that detect emergencies, for example fire. Lighting text based panels are also required to show the emergency situation and to guide workers toward the nearest stairs or elevator. With regard to the agents, new features which affect to the application must be considered. For example, people using wheelchairs move with a normal speed through the office but are unable to use the stairs. The simulation must also consider the concept of configurable emergencies. This is useful to simulate lots of scenarios in order to make the application more robust.

An important factor in Ubik, for its purpose, is the ability to operate with a large number of agents as well as a large space for these agents. For example, the largest already built skyscraper, Taipei 101, has 101 floors and a total construction area of 420,000 square meters. One of the main goals of the simulation platform MASON is supporting a large number of agents and being efficient at the same time[12]. Ubik, developed in MASON, has supported studies with simulations of more than 200,000 agents and 200 floors efficiently. How could be made a real test of an AmI application for 200,000 people?. What is the best way to evacuate 200,000 people from a building? We hope that MABS in general and Ubik in particular get closer to the answer. The following section is a proposal for analysis, understanding and debugging of Ubik, or any other simulation, through forensic analysis.

\section{DebUgGing SOCIAL SIMUlations FROM A FORENSIC PERSPECTIVE}

As noted above, the analysis phase of MABS has been traditionally performed in an exploratory and intuitive manner[4]. One of the weaknesses of MASON, and MABS platforms in general, is that they offer few facilities to monitor and debug the simulated models[12]. MASON offers the possibility of fixing inspectors in individual properties (of the model or of the agents) to be monitored / recorded / modified from the simulation. However, we miss many options as views and records of the artificial society as a whole.

Forensic analysis is the process of understanding, recreating, and analyzing arbitrary events that have previously occurred[11]. Serrano et al. [13] detail the creation of an infrastructure for forensic analysis in MAS. That technology can be brought to MABS. The key idea is to capture interesting elements of a simulation and to store them in a relational database (RDB). Once the RDB has captured the essence of a simulation, it is simple to make simplified representations of the stored data. These representations assist the analysis process. The field of MAS has already work with representations for these porpoises. For example, showing summaries of cooperation cores or similarities in the societies of agents[14]. Figure 1 shows the analyzer that records data in a RDB. It also shows a developer consulting that RDB and studying certain representations to analyze MABS.

Regarding how to capture elements of the simulation, it could be thought that it is something trivial. Calls to RDB can be simply programmed into the model or platform. This presents a major problem, this code would serve only for the specific model or the specific version of the platform. In addition, the logging code would be dispersed throughout the model or platform code. The solution in this proposal is the use of Aspect oriented programming (AOP)[8]. AOP can isolate the aspect of registering a MABS execution in order to treat it separately and in a modular manner. For now, our work is focused on the MASON platform. However, all the programmed aspects have equivalence in other platforms. Hence, replicating the results for other platforms is easy if they are distributed with the source code necessary to rebuild the platform as MASON does. Figure 1 shows how the analyzer can be used on different platforms. In this way we hope that our work is useful for the entire MABS community.

Regarding what elements are interesting to capture in a simulation, in a first approximation, it can be said that all those elements which were chosen to be monitored in the simulation are of interest. Here, the clarity of the platform code is a major factor. MASON code is extremely clear. Any method that starts with "get" in the code of an agent (implements the Steppable interface) or a model (implements SimState) returns a property that is displayed when this agent or model respectively is being inspected. Programming aspects to intercept all the calls to these methods is a trivial task. The body of the aspect store these properties in a RDB. Note that a complicated or confusing core of the platform would complicate extremely this stage. Once this basic approach has been covered, interesting elements present in all the simulations can be added to be registered. For example, the moment of creation of agents, their destruction or the interaction between them. The data 

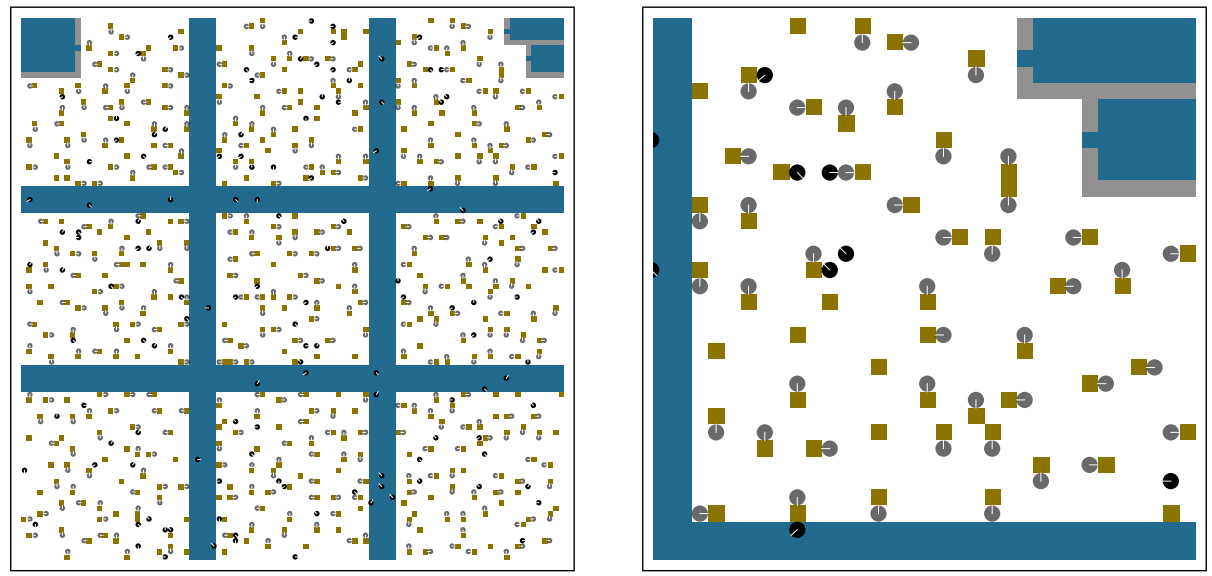

Fig. 2. On the left, a vectorial image of floor in Ubik with 500 agents. On the right, the zoom in the upper right corner of the floor

in RDB support simplified representations to be even more useful. In any case, the mere fact of having a RDB, which is a powerful tool for Querying, is a breakthrough that allows to do exploratory data mining, for example.

\section{CONCLUSIONS AND FUTURE WORK}

This paper set the basis of an infrastructure to study applications of ambient intelligence (AmI) which involve a large number of users. The infrastructure is a multi-agent based simulation (MABS) called Ubik. The paper also introduces an infrastructure to assist the analysis, understanding and debugging of a MABS extrapolated of multi-agent systems (MAS). The infrastructure consists of forensic analysis by aspect oriented programming (AOP).

The introduction to the paper has introduced how new techniques are needed to test AmI applications and how the MABS, a versatile technology which is at its very peak, can be used for that purpose. It has also pointed that MABS have opted for an analysis in an intuitive manner while the field of MAS has several proposals for such purposes. Related work has explained the classic approaches to test AmI applications, how MABS also serve for these purposes and the most interesting theories to analyze MAS. Section III has presented Ubik as a MABS to test AmI applications and the details and restrictions on those AmI applications. It also has given the example of a possible application of high social interest and which can be tested using Ubik, emergency assistance in an office building. Section IV has presented a proposal for analysis, understanding and debugging MABS. This proposal is based on forensic analysis and AOP. It has also shown that this infrastructure provides the MASON platform with a powerful analytical capacity.

Regarding future works, the first one is to extend Ubik to make it more descriptive. For example, with detailed configurations of space and agents expressed with ontologies. We also intend to include several AmI applications of social interest as emergency assistance has been included. The infrastructure to assist the analysis of MASON simulations also generates promising future works. The first one is a survey about representations to simplify the data of the forensic analysis.
Specifically, those representations that were successful in the development of MAS and may be exploitable in MABS. In general, we are interested in integrating our work with any technology that increases the usability of AmI applications or assists the analysis of MABS.

\section{ACKNOWLEDGEMENTS}

This research work is supported by the Spanish Ministry of Education and Science in the scope of the Research Project "Análisis, Estudio y Desarrollo de Sistemas Inteligentes y Servicios Telemáticos" through the Fundación Séneca within the Program "Generación del Conocimiento Científico de Excelencia".

\section{REFERENCES}

[1] John J. Barton and Vikram Vijayaraghavan. Ubiwise: A ubiquitous wireless infrastructure simulation environment, tech. report hpl2002-303. HP Labs, 2002, 2002.

[2] Rosaria Conte, Nigel Gilbert, and ao Sichman Jaime Sim Mas and social simulation: A suitable sommitment. In Proceedings of the First International Workshop on Multi-Agent Systems and Agent-Based Simulation, pages 1-9, London, UK, 1998. Springer-Verlag.

[3] Boris de Ruyter and Emile Aarts. Ambient intelligence: visualizing the future. In AVI '04: Proceedings of the working conference on Advanced visual interfaces, pages 203-208, New York, NY, USA, 2004. ACM.

[4] Alexis Drogoul, Diane Vanbergue, and Thomas Meurisse. Multiagent based simulation: Where are the agents? In Jaime S. Sichman, Francois Bousquet, and Paul Davidsson, editors, Proceedings of the Third International Workshop on Multi-Agent-Based Simulation MABS 2002, Bologna, Italy, LNAI 2581, pages 1-15. Springer Verlag, Berlin Heidelberg, July 2002.

[5] D. Lewis T. O’Donnell D. O’Sullivan E. O’Neill, M. Klepal and D. Pesch. A testbed for evaluating human interaction with ubiquitous computing environments. In TRIDENTCOM '05: Proceedings of the First International Conference on Testbeds and Research Infrastructures for the DEvelopment of NeTworks and COMmunities, pages 60-69. IEEE Computer Society, 2005.

[6] Michael Friedewald, Olivier Da Costa, Yves Punie, Petteri Alahuhta, and Sirkka Heinonen. Perspectives of ambient intelligence in the home environment. Telemat. Inf., 22(3):221-238, 2005.

[7] C.D. Kidd, R. Orr, G.D. Abowd, C.G. Atkeson, I.A. Essa, B. MacIntyre, E. Mynatt, T.E. Starner, W. Newstetter, et al. The Aware Home: A Living Laboratory for Ubiquitous Computing Research. LECTURE NOTES IN COMPUTER SCIENCE, pages 191-198, 1999.

[8] Ramnivas Laddad. AspectJ in Action: Practical Aspect-Oriented Programming. Manning Publications Co., Greenwich, CT, USA, 2003. 
[9] Sean Luke, Claudio Cioffi-Revilla, Liviu Panait, and Keith Sullivan. Mason: A new multi-agent simulation toolkit. In Proceedings of the 2004 Swarmfest Workshop, 2004.

[10] M.C. Mozer. The neural network house: An environment that adapts to its inhabitants. In Proceedings of the American Association for Artificial Intelligence Spring Symposium on Intelligent Environments, pages 110114, 1998.

[11] Sean Philip Peisert. A model of forensic analysis using goal-oriented logging. PhD thesis, La Jolla, CA, USA, 2007. Adviser-Sidney Karin.

[12] Steven F. Railsback, Steven L. Lytinen, and Stephen K. Jackson. Agent- based simulation platforms: Review and development recommendations. SIMULATION, 82(9):609-623, September 2006.

[13] Emilio Serrano and Juan A. Botia. Infrastructure for forensic analysis of multi-agent systems. volume Proceedings of the Programming MultiAgent Systems Workshop at AAMAS'08, 2008. Estoril, Portugal.

[14] Emilio Serrano, Jorge J. Gómez-Sanz, Juan A. Botía, and Juan Pavón. Intelligent data analysis applied to debug complex software systems. Neurocomputing, 72(13-15):2785 - 2795, 2009. 